\title{
Changes in the epidemiological profile of suicide in Hong Kong: a 40-year retrospective decomposition analysis
}

\author{
Chi-Ting Yang ${ }^{1}$ () Paul Siu Fai Yip ${ }^{2,3}$
}

Received: 4 February 2021 / Accepted: 15 June 2021 / Published online: 6 July 2021

(c) China Population and Development Research 2021

\begin{abstract}
The suicide rate in Hong Kong has increased significantly over the past four decades. Population subgroups such as the elderly or economically-distressed are reported to be more vulnerable than others to suicidal behaviors, while changing suicide methods (such as charcoal burning which emerged in 1998), has also contributed significantly to increasing suicide rates. However, the extent of the contribution of different factors to changes in suicide rate remains unclear. This paper reported on a decomposition analysis of the epidemiological profile of suicide in Hong Kong between 1976 and 2015, specifically considering factors underlying the increasing suicide rate over this period. Completed death registry information was available from the Census and Statistics Department of the Hong Kong SAR for this investigation. We compared absolute and relative contributions of gender, age and suicide method to rate changes over time. Changes in suicide rate were generally underpinned by more than one factor. Population aging in a rapidly-aging city contributed significantly to suicide rate increases, whilst jumping from a height had the greatest influence on rate changes throughout the study period. Suicides by male aged 25-34 years and 45-54 years were more likely to be triggered by economic factors, compared with the other gender-age subgroups. The decomposition approach provided a comprehensive understanding about how socioeconomic factors and suicide methods interacted to influence over-time suicide patterns. This research supports development of more focused suicide prevention measures to reduce suicide rate.
\end{abstract}

Keywords Suicide $\cdot$ Hong Kong $\cdot$ Decomposition analysis $\cdot$ Epidemiological trend

Paul Siu Fai Yip

sfpyip@hku.hk

1 Department of Statistics and Actuarial Science, The University of Hong Kong, Hong Kong, China

2 Hong Kong Jockey Club Centre for Suicide Research and Prevention, The University of Hong Kong, Hong Kong, China

3 Department of Social Work and Social Administration, The University of Hong Kong, Hong Kong, China 


\section{Introduction}

After being a British colony for over 150 years, Hong Kong (HK) was returned to the People's Republic of China in 1997, whilst maintaining a separate governing and economic system under the principle of "one country two systems" (The Government of Hong Kong Special Administrative Region, 2020). HK has undergone a series of structural changes in transitioning first from a small fishing village to a manufacturing city in the 1960-1980s, and then to a service-based economy by the early 1990s (Carroll, 2007; Dodsworth \& Mihaljek, 1997). It is now a global financial centre and shipping hub. Immediately after the handover to China, HK experienced several severe crises including the 1997 Asian financial turmoil, the outbreak of H5N1 Avian flu followed by the 2003 SARS epidemic (Carroll, 2007; Lee, 2006). The ongoing debate about sovereignty has concentrated mostly on democratic development and the future political system in the post-handover period (Lam et al., 2012; Ma, 2007). The decision made by the central government in implementing nominee pre-screening of Chief Executive election triggered the 2014 pro-democracy Occupy Movement (Kaiman, 2014). The proposed Anti-Extradition Law Amendment Bill 2019 (Anti-ELAB) sparked the most substantial social unrest since the handover of 1997 (Teo \& Fu, 2021). Political and social unrest in the territory in 2019 introduced a high degree of uncertainty, complexity and ambiguity that inevitably triggered adverse mental wellbeing (Ni et al., 2020). The Covid-19 pandemic in 2020 has also caused considerable and ongoing anxiety in the community (Yip \& Chau, 2020). The socioeconomic and political situations are still undergoing rapid changes at present.

Hong Kong is famous for its densely populated city with a population of over 7.4 million people living in less than a 1,000 square kilometer area. There reflects occupation of only $7 \%$ of Hong Kong land, with more than $80 \%$ residents living in high-rise buildings (Task Force on Land Supply, 2018). Coupled with these changes, HK experienced two periods of significant population growth (1976-1981 and 1993-1997) and a concurrent demographic change, particularly in population aging, resulting from low fertility and higher life expectancy (Wong \& Yeung, 2019). While it took a century for France to double its population of older adults, it only took 30 years for the same population outcome to occur in HK (from $7.0 \%$ in 1983 to $14.2 \%$ in 2013) (World Health Organization [WHO], 2020).

Anonymized information on death by suicide is available publicly in HK for people aged 15 or above. There were 919 cases of death by suicide recorded in 2018 in HK, an estimated suicide rate of 12.3 per 100,000. This reflects approximately 9.3 deaths by suicide per 100,000 people, a figure that was lower than the age-standardized global rate in the same time period (11.2 per 100,000) (Hong Kong Jockey Club Centre for Suicide Research \& Prevention [CSRP], 2020). The suicide rate in HK has experienced dramatic ups and downs in the past four decades; reaching its highest point in 2003 (18.6 per 100,000), although the rate has since reduced and levelled off to 12.0 per 100,000 in 2015. Jumping from a high place has traditionally been the most common suicide method in HK (Hau, 
1993; Yip, 1997). Around the early 2000s, about $46 \%$ of the increase in the suicide rate was explained by the increasing age profile of people dying by suicide (Yip et al., 2003). Older adults in HK, as in many other Asian countries, have a higher suicide rate than the population average in Western countries (Chen et al., 2012). Moreover, the high suicide rate in HK in the early 2000s had a significant socio-economic component, notably associated with the Asian financial crisis of 1997-1998 and global economic downturn with the fall of Lehman Brothers Holdings Inc. (Chang et al., 2014; Law et al., 2011). This contributed to significant levels of unemployment in HK and for many, a loss of face and credibility within their family and community. It is not surprising that the unemployed was over-represented among suicide deaths, while the emergence of a new suicide method in 1998, charcoal burning, has also measurably increased suicide rates (Yip et al., 2003). However, the extent of the contribution of underlying factors to the HK suicide rate remains unclear (Law et al., 2011).

In line with Moksony's composition theory (1990), the discrepancy in suicide rates between nations might be explained by national population differences in the proportion of those at-risk of suicide at any one point in time. The rapidly aging HK population, and the concerning frequency of suicides by older people in HK, are cogent reasons to seek a better understanding of $\mathrm{HK}$ suicide patterns and predictors, so that suicide prevention policies can target the most vulnerable. This paper reports on a decomposition analysis of the epidemiological profile of suicide in $\mathrm{HK}$ between 1976 and 2015. We aimed to estimate the contributions made to changes in HK suicide rates over this time by social-demographic variables, suicide method and economic factor.

\section{Materials and methods}

\subsection{Data sources}

Completed records on suicide deaths between 1976 and 2015 was available from the registered deaths database held by the Census and Statistics Department of the Hong Kong SAR (from 1st July 1997 and onwards, the Government of the HK Special Administrative Region of the People's Republic of China). Following the International Classification of Diseases and Related Health Problems (ICD), all reportable deaths with an external cause code from E950 to E959, for 1976-1978 under ICD-8 and 1979-2000 under ICD-9 (WHO, 1978), were classified as suicide. From the years 2001 onwards, the classification of suicide deaths was based on updates of the ICD-10 with coding from X60 to X84 and X870 (WHO, 1992). We followed the practice that those suicide decedents with external cause code as E952 (for cases from 1976 to 2000) and X67 (2001 and onwards) fulfilling the definitions of "intentional self-poisoning by and exposure to other gases and vapors" were categorized as charcoal burning (CO) poisoning. Previous research reported that more than $90 \%$ of deaths in this category were charcoal-burning suicides in HK and Taiwan (Lin et al., 2008). 
Independent variables of gender, age, date of death, cause of death, occupation, place of residence and marital status were available from the death records. Age was considered in subgroups of 15-24, 25-34, 35-44, 45-54, 55-64 and $>=65$ years. Four methods of suicide, namely hanging, jumping, poisoning in general (poisoning), and charcoal-burning poisoning (charcoal burning) accounted for more than 92\% total suicides over this study period. Thus all other suicide methods were grouped together and assessed as "other". We obtained official mid-year population estimates from Census and Statistics Department where the data was stratified by gender and age (in five-year age bands).

\subsection{Statistical methods}

Changes in suicide trend annually throughout the study period were first examined using Joinpoint regression analysis (rates of change determined at $1 \%$ level of significance) (National Cancer Institute [NCI], 2020). Five significant time points of change were identified (Fig. 1). The contributions of gender, age and suicide method factors to the year-specific suicide rates of 1976, 1982, 1985, 1999, 2003, 2006 and 2015 were consequently examined.

Decomposition analysis was employed to provide an empirical assessment of how specified factors contributed to the rate changes in suicide over the 40-year period. We followed the two-factor method as proposed by Retherford-Cho (1973)

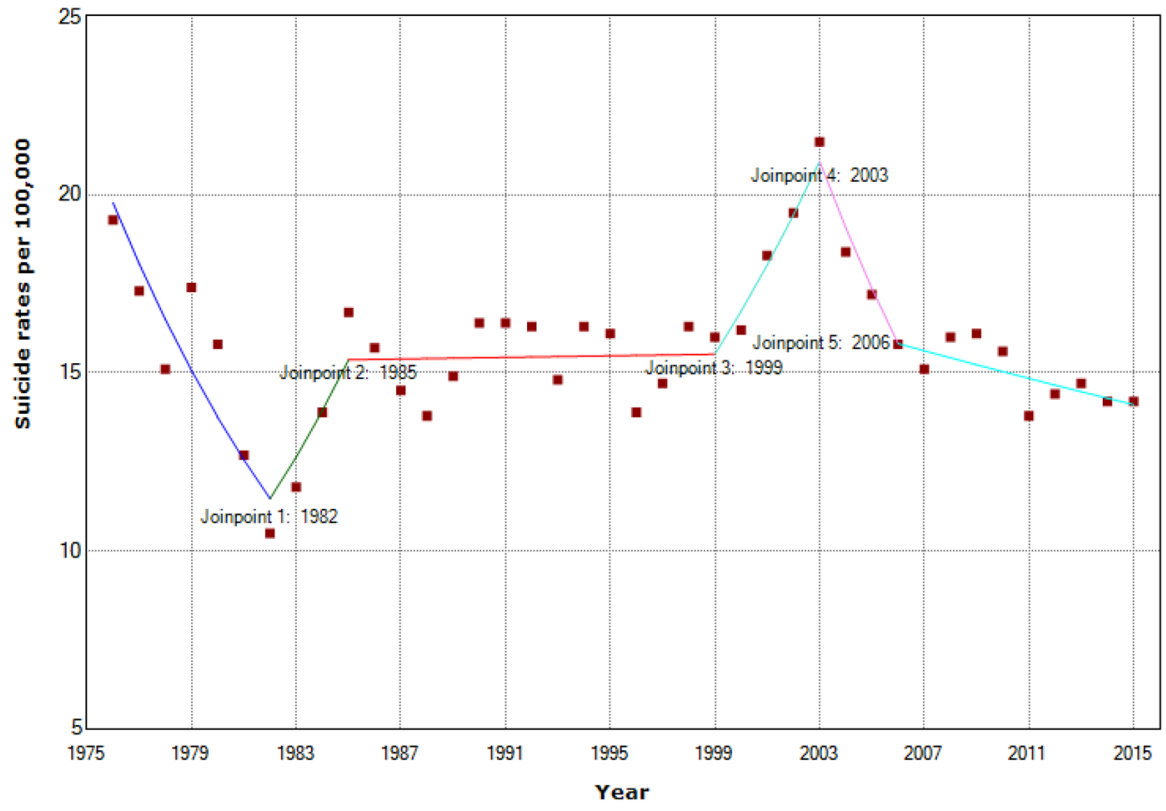

Fig. 1 Trends of suicide rates during the period of 1976-2015 in Hong Kong (aged 15 or over) where five significant turning points were identified by Joinpoint regression analysis at the $1 \%$ level of significance 
and decomposed the rate change in suicide deaths into three factors: (1) the change due to gender difference; (2) the change due to age composition of population by gender; and (3) the change due to the suicide rate using different self-harm methods, specifically by gender and age composition. This approach identified absolute and relative contributions of factors to the corresponding change of suicide patterns at key time points throughout the 40 year study period.

Kendall's rank correlation coefficients were calculated to evaluate whether suicide rates by gender and age group were specifically tied to the changing unemployment pattern in HK throughout the study period (SAS Institute Inc. [SAS], 2016). We assessed the ordinal association based on the number of concordant and discordant paired observations for each sentinel year in which rate change occurred. The technique of min-max normalization was applied by rescaling both measurements into a range of 0 and 1 , subtracting the minimum value from each original data then by dividing the corresponding difference between the maximum and minimum values. This approach performs better in the ordinal analysis while all relationships in the data are preserved.

We employed Joinpoint Regression Programme software (Version 4.8.0.1, Statistical Methodology and Applications Branch, Surveillance Research Programme, National Cancer Institute, Bethesda, Maryland, USA) and SAS version 9.4 for our analysis.

\section{Results}

\subsection{Overview of suicide rates between 1976 and 2015}

Figure 1 describes the change of HK suicide rate per 100,000 over the study period. There was a decrease in suicide rate from 1976 until 1982 (from an initial high of 19.3 to 10.5 per 100,000$)$. The rate increased to 16.7 per 100,000 in 1985 , then plateaued between 1985 and 1999. The second increase in suicide rates occurred between 1999 and 2003, with a 7.8\% annual growth until it hit a historical high of 21.5 per 100,000 in 2003 . The rate has subsequently fallen to 15.8 per 100,000 in 2006 and further to 14.2 per 100,000 in 2015 .

\subsection{Gender and age contributions}

There were 31,511 suicide deaths (12,317 females and 19,194 males) which fulfilled the selection criteria (aged 15 or above) over the study period. Compared with females, males had an overall higher rate of suicide (Fig. 2) though both exhibited similar patterns of change over time. The suicide rate increased steadily with age (Fig. 3). Nearly half the suicide deaths $(48.5 \%)$ occurred by jumping from high places, and nearly one-third (28.1\%) died by hanging (Fig. 4). After the first case of charcoal-burning poisoning death was widely reported in local media in October 1998 (Chan et al., 2003), there was an increase in copycat suicides that accounted for one tenth (9.9\%) of the total deaths in our study. 
158

C.-T. Yang, P. S. F. Yip

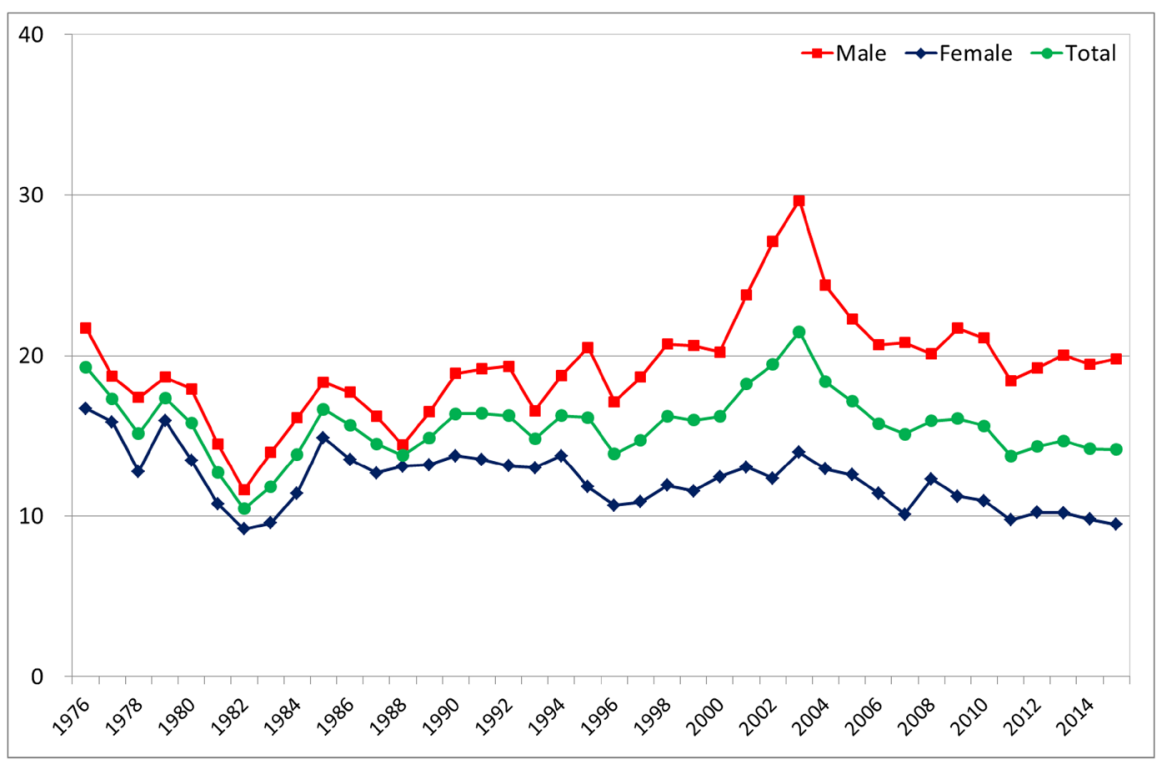

Fig. 2 The trend of suicide rate during the period of 1976-2015 in Hong Kong by gender (aged 15 or over)

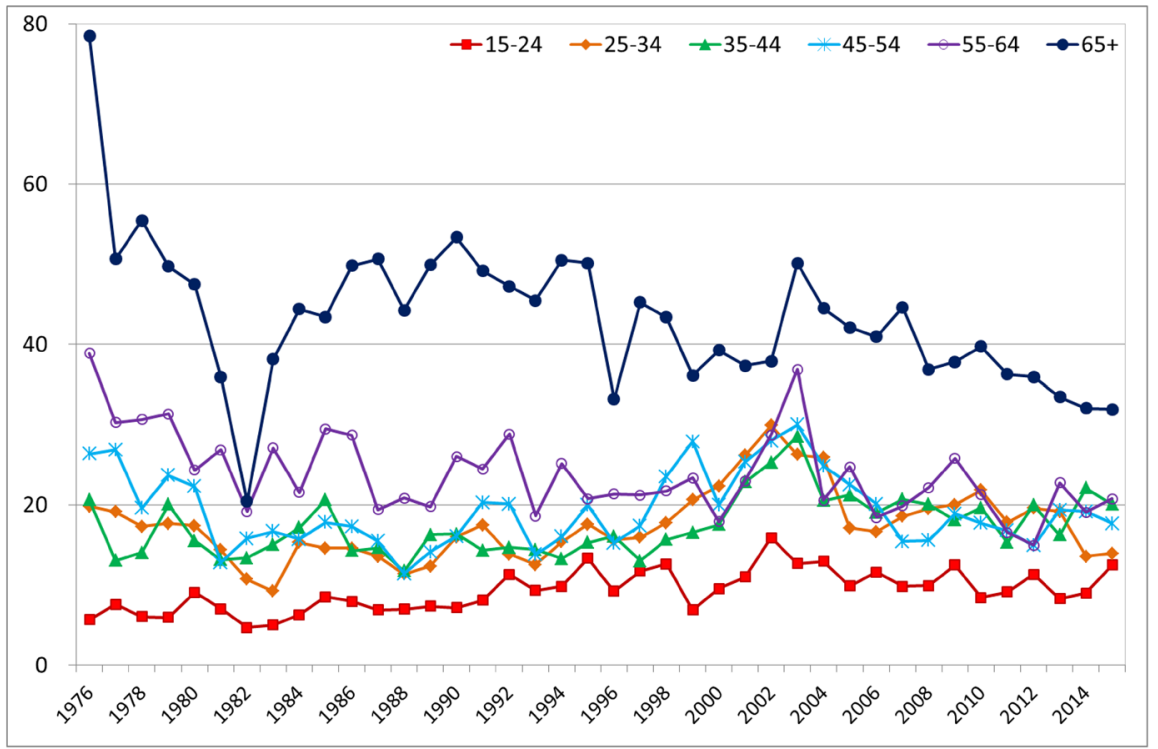

Fig. 3 The trend of suicide rate during the period of 1976-2015 in Hong Kong by age group (aged 15 or over)

Springer 


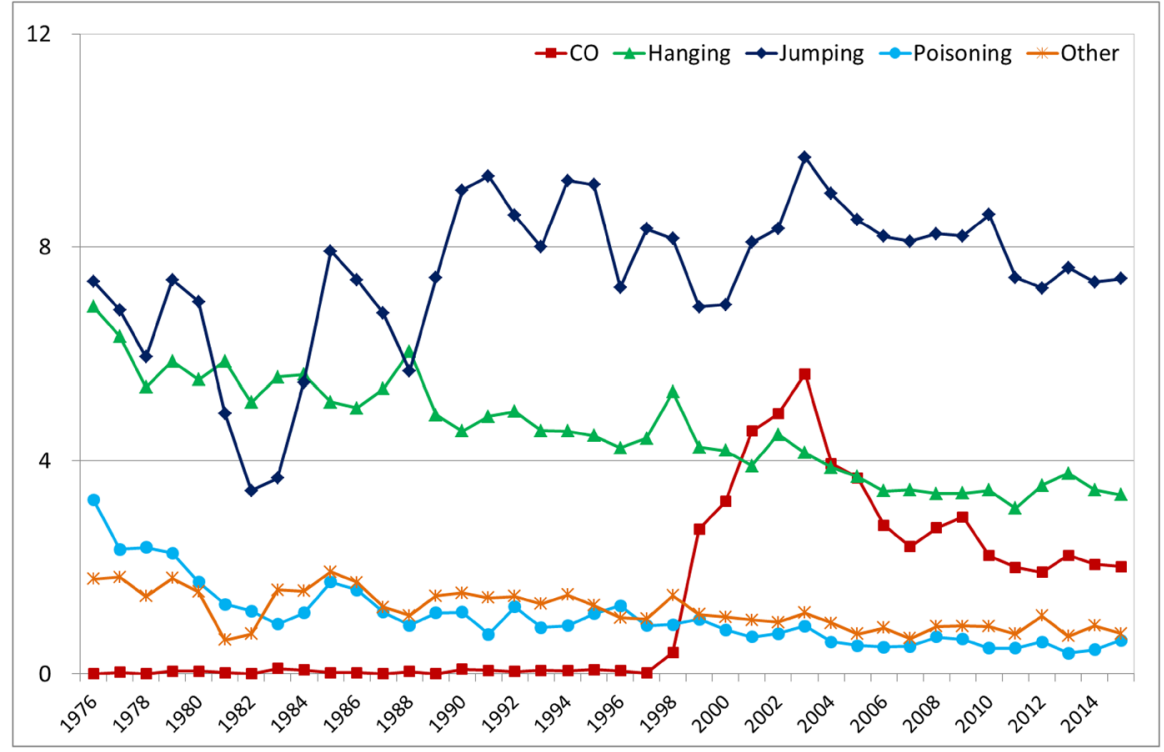

Fig. 4 The trend of suicide rate during the period of 1976-2015 in Hong Kong by method use (aged 15 or over)

\subsection{Decomposition of the rate change in suicide between 1976 and 2015}

Table 1 reports the contribution of gender, age and method use to the rate change in suicide for the six time periods of interest. A negative value implies fewer people taking their own lives during that period.

\subsubsection{Rate change in suicide 1976-1982 (reduced by - 8.8 from 19.3 to 10.5)}

Fewer suicides occurred by jumping from high places was the key contributor to the rate decline during that period (males and females together explained $47.2 \%$ ), and this change was particularly apparent among males aged 45 years and older (Fig. 5). Fewer males died from hanging, particularly those aged $65+$ years, which was a key driver for the decreasing rate $(15.9 \%)$ while male suicides by poisoning explained $13.4 \%$ of the rate fall for the period. However, an increase in the proportion of decedents aged 25-34 years (8.7\%) and $65+$ years $(6.7 \%)$ led to the over-representation of these age groups in the suicide rate, which in turn, drove the overall suicide pattern up.

\subsubsection{Rate change in suicide 1982-1985 (increased by 6.2 from 10.5 to 16.7 )}

The sharp reversal of the suicide rate in 1982-1985 was mainly due to decedents jumping from high places (females $37.3 \%$ and males 33.0\%) (Fig. 5). The impact of other methods did not contribute much to the overall increasing rate. Part of 


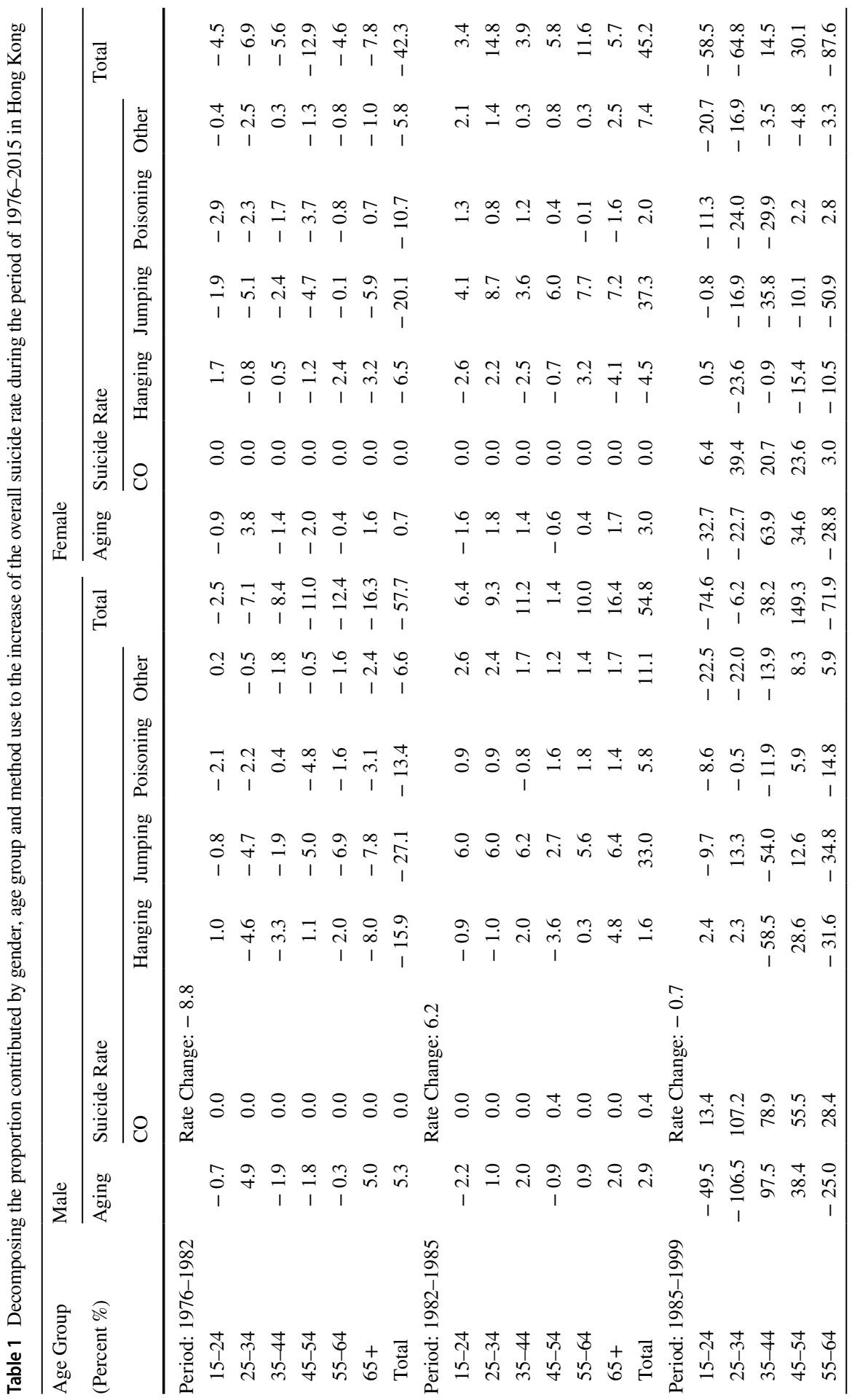




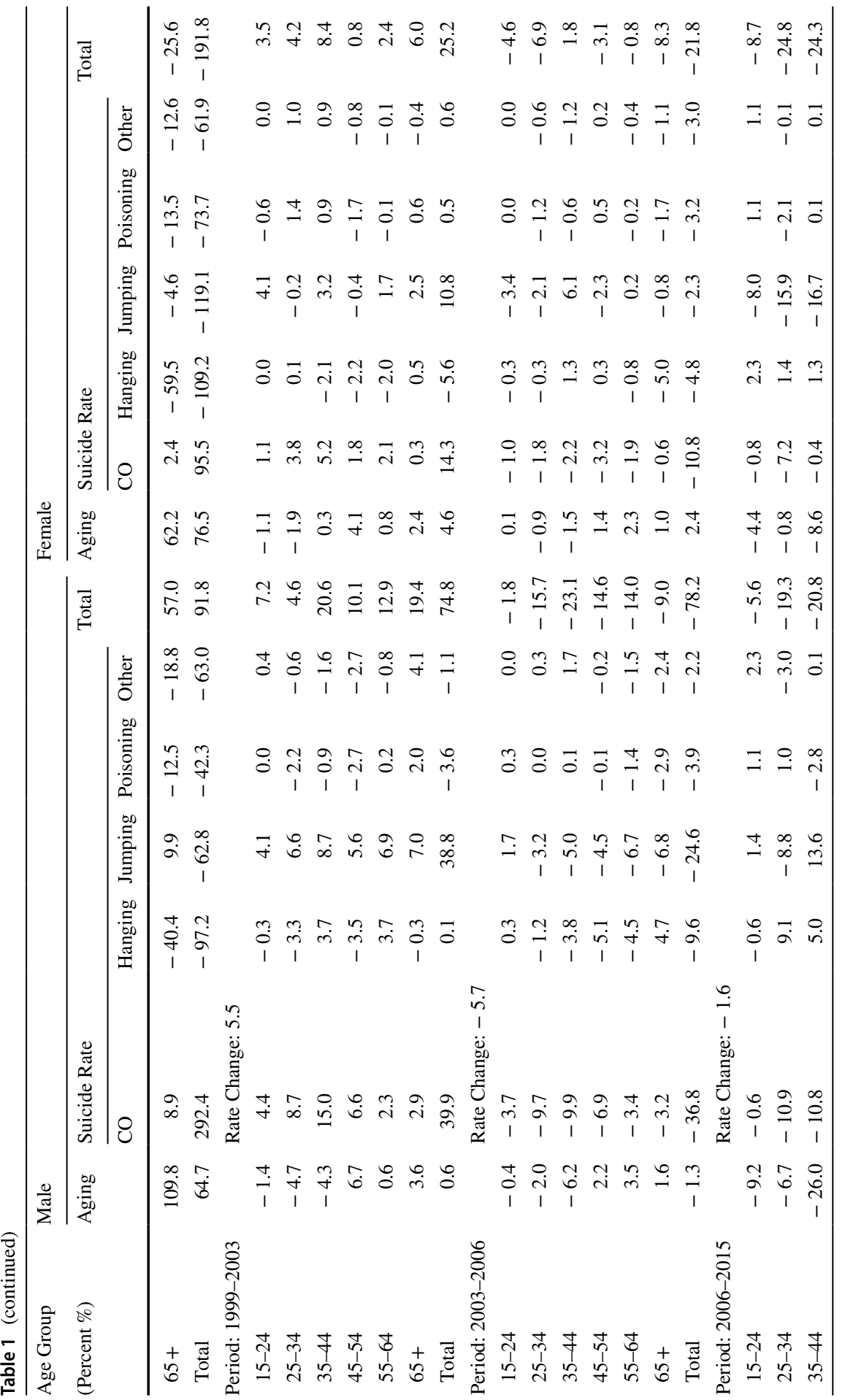




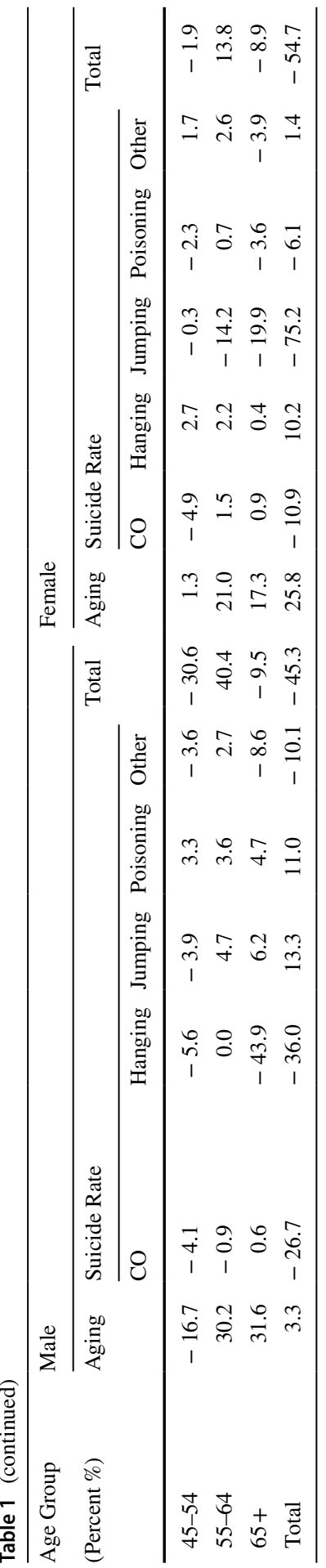

\section{글 Springer}



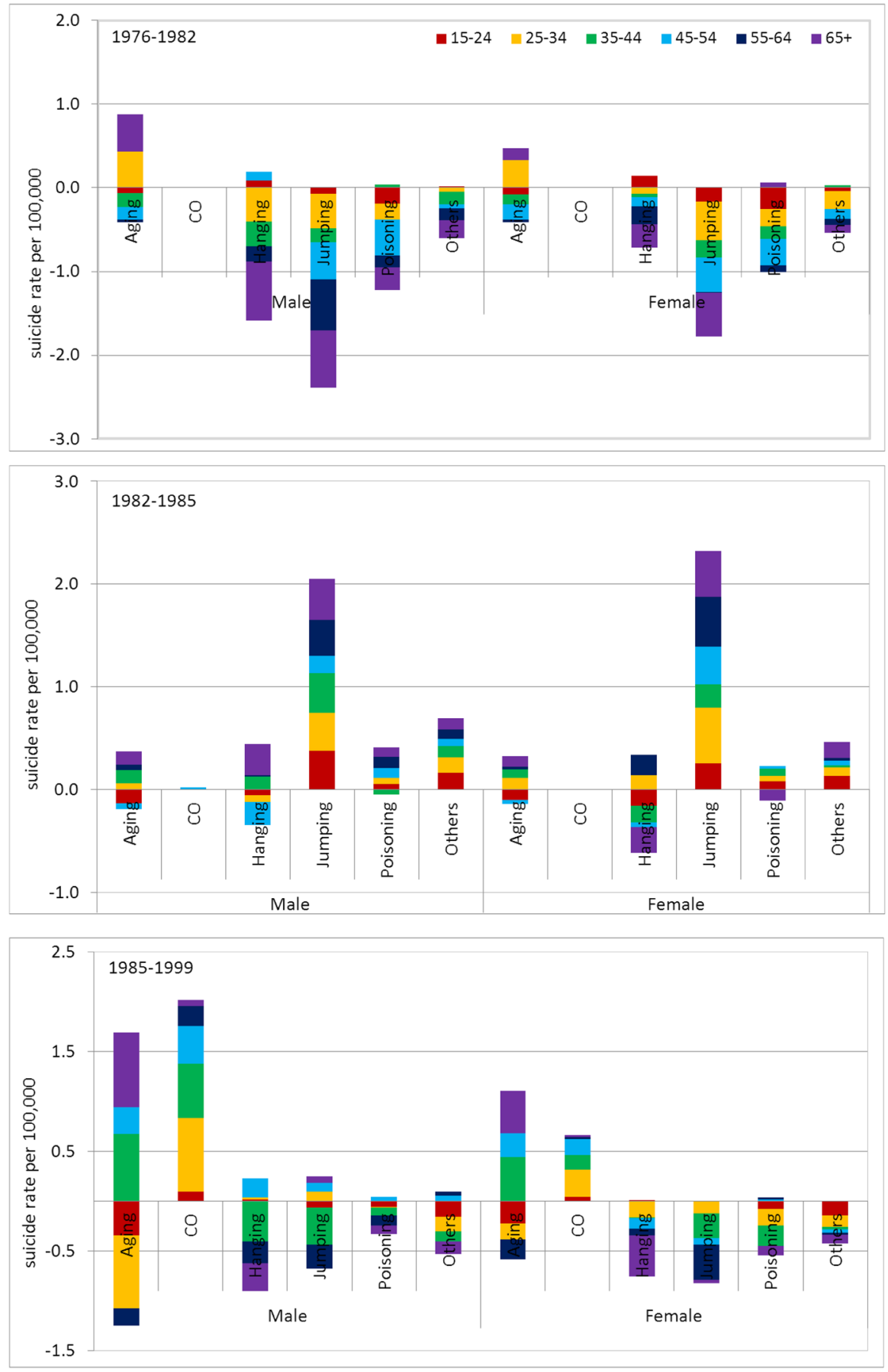

Fig. 5 The absolute contribution by gender, age group and method use to the increase of the overall suicide rate during the period of 1976-1982, 1982-1985 and 1985-1999 in Hong Kong 
the reason for rate increase during this period could also be explained by the change in age composition reflected in the age groups of 35-44 and 65+years. HK had officially entered the era of an aging society by 1983 and the population growth in older adults was solely responsible for the rate increasing by $3.7 \%$ in this period.

\subsubsection{Rate change in suicide 1985-1999 (decreased by - 0.7 from 16.7 to 16.0)}

The rate of suicide was stable in this period, with only mild fluctuations over the next 14-years. However, the non-significant change (- 0.7 per 100,000$)$ undermined some of the important structural variation in suicide rate by method and by age group during the period. The decomposition analysis showed that all traditional methods recorded significant reductions in the period that all together explained about $629.1 \%$ of the rate change. The most obvious reduction was contributed by females jumping from high places (119.1\%). Charcoal-burning suicides appeared during this period as a new method that attracted much attention in the community. Deaths by this method climbed from one case in 1997 to 22 cases in 1998, and further to 148 cases in 1999. Against the overall rate decline, an increasing number of decedents adopted charcoal-burning as a method of suicide (387.9\%) which impacted mostly on males aged 25-34 and 35-44 years. It seems that there was a displacement effect of traditional methods (jumping and hanging) by the emergent method (charcoal burning). Moreover, the aging effect was very apparent as evidenced by the major growth due to the impact of older adults $65+$ years $(172.1 \%)$ followed by the middle-aged cohort 35-44 years (161.4\%). The impact of changes in age composition together with the emerging method of charcoal burning almost cancelled out the reduced contributions by traditional suicide methods for the period.

\subsubsection{Rate change in suicide 1999-2003 (increased by 5.5 from 16.0 to 21.5)}

Many people lost their jobs and suffered financial difficulties due to the Asian economic turmoil occurring in the summer of 1997. This was reflected by an increase in suicide cases which rapidly grew within a few years to a historical high of 21.5 per 100,000 in 2003, the time when HK was devastated by the SARS epidemic. Male suicides by charcoal-burning (39.9\%), particularly for middle-aged (35-44 years), was the key driver for the growth for this period, followed by males jumping from high places (38.8\%) (Fig. 6). A similar pattern could be observed among female suicides but mild mannered. Also, the change of age composition among people aged 45-54 years and older adults were key contributors to the rate increment between 1999 and 2003 (10.9\% and 6.1\%). Of note is that male gender recorded three quarters positive contribution to the overall rate change for the period after taking all factors into account.

\subsubsection{Rate change in suicide 2003-2006 (decreased by - 5.7 from 21.5 to 15.8)}

Health and economic activity in HK gradually got back on track after 2003, reflected in a lower unemployment rate and an improvement from the suicide epidemic by 

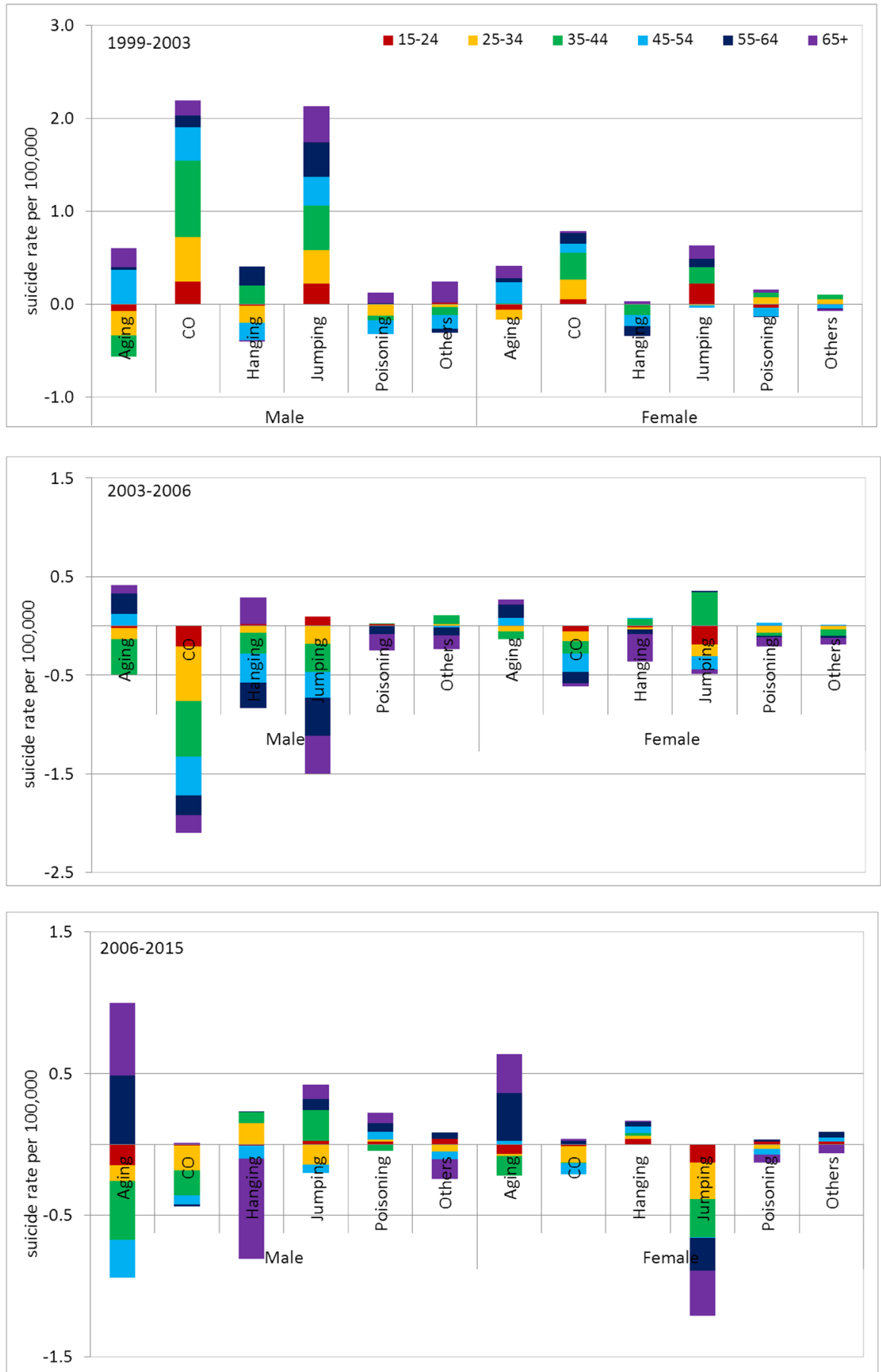

Fig. 6 The absolute contribution by gender, age group and method use to the increase of the overall suicide rate during the period of 1999-2003, 2003-2006 and 2006-2015 in Hong Kong 
comparing with the previous period. The rate of suicide dropped from the historical high in 2003 to 15.8 per 100,000 in 2006 . Male suicides by charcoal-burning contributed the most (36.8\%) for this period and the improvement was more apparent amongst the young and middle age groups (Fig. 6). Meanwhile, there was an obvious decline in the percentage of males jumping from high places (24.6\%), mostly reflecting adults aged 55 years and older. It seems that male gender contributed more to the decline of the suicide rate during the period than any other subgroup, perhaps as they were more sensitive to the economic recovery.

\subsubsection{Rate change in suicide 2006-2015 (decreased by - 1.6 from 15.8 to 14.2)}

The rate of suicides continued to decrease between 2006 and 2015 but at a gentle pace. Females jumping from high places explained $75.2 \%$ of the downward trend followed by the males hanging themselves (36.0\%). Another key contributor was male suicides by charcoal-burning (26.7\%) reflected most in those aged 25-34 years and 35-44 years. In 2013, HK was officially acknowledged as an aged society, with over $14 \%$ of its population being classified as elderly (Wong \& Yeung, 2019). Against the overall decline in suicides, the population aging amongst people aged 55 years and older drove the upward trend of suicide (Fig. 6).

\subsection{Impact of unemployment status on suicide rate}

HK started the 40-year study period (in 1976) with a relatively high unemployment rate $(5.1 \%)$, which fell to $3.6 \%$ in 1982 and further to $3.2 \%$ in 1985 . The economic downturn brought by the 1997 Asian financial crisis was responsible for increasing the unemployment rate to $6.2 \%$ in 1999 and then to a record high of $7.9 \%$ in 2003 . Economic recovery in $\mathrm{HK}$ began in late 2003, as evidenced by the ongoing drop in the unemployment rate ( $4.8 \%$ by the end of 2006 to $3.3 \%$ in 2015). Table 2 reports the Kendall rank correlation coefficient by gender and age group, specifically measuring the statistical dependency between the changing suicide rate and the changing unemployment pattern in $\mathrm{HK}$ at different time points. A strong ordinal association was observed with suicides by males aged $25-34$ years $(0.71, p=0.02)$ and males aged $45-54$ years $(0.71, p=0.02)$, suggesting the impact of unemployment in middle-aged male subgroups.

Table 2 Kendall rank correlation coefficient by gender and age group between the changing suicide rate and the changing unemployment rate during the period of 1976-2015 in Hong Kong at 5\% level of significance

\begin{tabular}{lcccccc}
\hline Age group & $15-24$ & $25-34$ & $35-44$ & $45-54$ & $55-64$ & $65+$ \\
\hline $\begin{array}{l}\text { Male } \\
\text { Tau }\end{array}$ & 0.14 & $\mathbf{0 . 7 1}$ & 0.10 & $\mathbf{0 . 7 1}$ & 0.14 & 0.24 \\
$\quad p$-value & 0.65 & $\mathbf{0 . 0 2}$ & 0.76 & $\mathbf{0 . 0 2}$ & 0.65 & 0.45 \\
$\begin{array}{l}\text { Female } \\
\quad \text { Tau }\end{array}$ & 0.43 & 0.33 & 0.05 & 0.14 & 0.14 & 0.05 \\
$p$-value & 0.18 & 0.29 & 0.88 & 0.65 & 0.65 & 0.88 \\
\hline
\end{tabular}




\section{Discussion}

Suicide ranked sixth in the leading cause of deaths in HK, accounting about 3\% of all deaths each year (Yip \& Zheng, 2021). The important implication of suicide in $\mathrm{HK}$, from a public health perspective, is the burden on society of years of productive life lost, particularly by middle-aged decedents, and this could be a useful indicator of economic and health performance in HK. This paper is the first retrospectively to analyze 40-years of the HK suicide epidemic using a decomposition statistical approach. This analysis provides a clearer picture of the impact of gender, age, suicide method and unemployment on suicide rates, as well as how these factors work together to influence HK suicide patterns. The specific contribution of gender and suicide methods to changing suicide rates varied across six time periods, although the age influence played an important role throughout the time period.

Concurring with previous studies on suicide in HK (Hau, 1993; Yip, 1997; Yip et al., 2003), jumping from high places was the most common suicide method between 1976 and 2015 and the key contributor to fluctuations in the overall rate in our study. The prevalence of jumping might be explained by its accessibility and lethality given that HK is the world's densest city, where most people live in multistory buildings. Installing barriers such as platform screen doors have been found to be effective suicide prevention strategies in HK railway stations, thus adopting similar community-based suicide prevention approaches for high-rise buildings may be similarly effective (Lai et al., 2020; Law et al., 2009). Experiences of restricting access of a suicidal person to charcoal or displaying warning labels on charcoal packs appear to have been another effective suicide prevention model in the community (Wu et al., 2012; Yip et al., 2010). Guidelines for media reporting on suicide incidence and method have also reduced the copycat effect in vulnerable groups (Chan et al., 2003). The reduction in charcoal-burning suicides for the period of 2003-2006 and 2006-2015 appears to support the effectiveness of such community-based suicide intervention strategies in HK.

Unemployment is a key economic indicator that measures the efficiency and effectiveness of an economy, and it also plays a role in explaining changes in suicide rate (Kuroki, 2010; Law et al., 2011). Concurring with other studies (Chang et al., 2014; Law et al., 2011), our findings showed that suicides of male aged 25-34 years and 45-54 years were much more likely following the trend of economic factors when compared with other subgroups in the study period. The dependency results from the non-parametric model explained the high incidence of suicide deaths for working-aged men during economic downturns. The consecutive fall of suicide rates during the periods 2003-2006 and 2006-2015 in our study, therefore, reflected the ongoing economic recovery.

For males aged 35-44 years, the changes in their suicide rate was not in line with the unemployment rate over the study period $(p=0.76)$. The first peak found in 1982-1985 (from 13.4 to 20.6 per 100,000) was likely to be associated to economic restructuring when most of the manufacturing production lines in HK were re-located to the southern part of China. The HK economy has been 
gradually integrating with southern China acting as "regional economy hub" after the Open-Door policy and economic reforms of China in 1979 (Wong, 2016). Such deindustrialization became significant after the early 1980s. The challenges from economic restructuring and the importation of migrant workers still threatened local working-aged people although HK maintained at a low unemployment rate in 1980s. The second peak occurred in 1999-2003 (from 16.5 to 28.6 per $100,000)$, which was known to be related to several macro environmental events including the Asian financial crisis and the SARS outbreak. The new method of charcoal-burning mainly attracted unmarried, economically-active middle-aged males (aged 35-44 years) who were more likely to be experiencing financial difficulties (Yip et al., 2003). The suicide rate among this subgroup remained high, at 20.0 per 100,000 in 2015 despite the ongoing economic recovery which was observed after 2003. This finding was specific for men, as the rate change among female suicides in our study was not related to the unemployment rate.

Many studies suggested that males are more sensitive to social-economic changes, which potentially explains the generally higher rate of suicide in men during periods of social turmoil (Chen et al., 2020). The risk exposure in males compared with females in suicide mortality has been suggested to be a complex biological vulnerability related to a series of gender-specific sociocultural stressors (Chung et al., 2016). On its own, the factor of unemployment may not be enough to explain the discrepancy in suicide rates between genders, particularly in such a developed society as HK. In fact, HK suicides have a very different socio-economic profile from those in Western countries, and the HK pattern has undergone significant changes over the past four decades. Marriage postponement or non-marriage has increased dramatically in recent years $32.4 \%$ males and $28.0 \%$ females had never been married in 2016) while the crude divorce rate grew from 1.1 cases per 1000 in 1991 to 2.3 per 1000 in 2016 (Hong Kong Monthly Digest of Statistics [HKMDS], 2017). The importance of family concepts and the socialization functions of family which once protected against suicide are gradually disappearing in HK society (Chung et al., 2016). Cross-border marriages between Mainland Chinese and HKese have also increased dramatically (from 1.6\% out of the total registered marriages in 1991 to $34.7 \%$ in 2016) as a result of frequent social interactions and the high economic integration between two places after the 1997 handover (HKMDS, 2017). Changes in marital distribution together with the societal disparity between two places put increasing pressure on social support and welfare services in $\mathrm{HK}$, and this poses a significant challenge to the work of suicide prevention (Chen \& Yip, 2019; Kan \& Ma, 2008).

Hong Kong is currently topping life expectancy rankings in the world, with average age of 82.2 years for males and 88.1 years for females as of 2019 (Center for Health Protection, 2021). This implies an increase in the proportion of elderly in the population. Given that HK was acknowledged as an aged society in 2013, longlife expectancy poses a challenge not just for competing resources to support elderly people in the community, but also for its contribution to increasing elderly suicides. As indicated by our analysis, the change in age composition could solely underpin the upward trend of suicide rates, suggesting that the impact of aging in the community may be more alarming than initially thought. The pace of population aging in 
HK will be more pronounced in the next twenty years, as the proportion of elderly in the HK population is expected to grow from $16.6 \%$ in 2016 , to $31.1 \%$ in 2036 , with a concomitant decrease in the percentage of people younger than 65 years (HKMDS, 2017). Moreover, the support ratio of young people for elderly (the number of working individuals aged 15-64 for every elder) will fall from nearly four persons (3.93) in 2018 , to an estimated 1.83 in 2038 , demonstrating that there will be a much heavier burden of economic responsibility on future generations (Wong \& Yeung, 2019).

Table 3 Key suicide prevention programme and project during the period of 2001-2018 in Hong Kong

Year Key Suicide Prevention Programme and Project in Hong Kong

2001 Live Life! Joint Project on Prevention of Elderly Suicide Suicide Prevention Service for Elderly Hotline (2382-0881)

2002 The establishment of HKJC Centre for Suicide Research and Prevention

The establishment of Suicide Crisis Intervention Centre (24-h Hotline)

The establishment of Central Suicide Information System

The establishment of Life Education Centre

Suicide Prevention Service for Youths-SHKP Operation Sunshine Programme

Elderly Suicide Prevention Programme-Support elderly people with suicidal tendency at psychogeriatric fast track clinics

2004 Little Prince is Depressed-School-based mental health enhancement programme

Live the Rainbow Programme-One stop services including counselling, small groups and continuous support service in which the survivors will be accompanied in their difficult moments

2006 Barbecue charcoal packs removal project in Tuen Mun

Outreach Befriending Service for the Suicidal Elderly

2007 "Don't Qua" Elderly Visit Programme-The first large-scale seasonal volunteer service programme

2008 Suicide Prevention on neT and Web Engagement Project—Suicide prevention services on internet BLESS Programme-Services for survivors of suicide loss

2010 Revitalizing the Elders Project—Promote positive life attitudes in the community

2011 Filling Lives with Joy for Teenage Life Education Programme

Problem-solving Skills Training and Mentorship Programme for Adolescents

2012 Action for Happiness - Individual Activity Plan for Depressive Elderly—Promote positive life attitudes in the depressed elderly through tailoring activity plans

Gatekeeper Training Project for Youth Suicide Prevention Project

Professor Gooley \& the Flame of Mind Programme-A web-based mental health programme for adolescents with parental involvement

2013 KidMatters Programme-A Web and School-based approach to mental health promotion among children in primary schools

2015 Quality Education Fund Thematic Network on Developing Students' Positive Attitudes and Values

Recommendations on Suicide Reporting \& Online Information Dissemination for Media Professionals

2016 WeCare Fund for Student-Initiated Youth Suicide Prevention Projects

Family Support for Elderly Mental Health

Youth Link Hotline (2382 0777)—Support youth aged below 24

2018 Chat Point in 2018 - A cross-platform suicide prevention and emotional support service 
Suicide prevention efforts made by the HK Government, and non-governmental organizations (NGOs) together with research undertaken by HK universities have possibly contributed to the overall decrease in suicide rates in past two decades (Table 3) (CSRP, 2020). Learning from other countries, appropriate policies and programs from public health perspectives can keep older adults healthy and independent, so they can continue to contribute to society (WHO, 2020). The findings from this decomposition analysis give policymakers a better understanding of the implications and consequences of aging, and their possible impact towards suicidal behavior in HK.

\section{Limitation}

Significant contributions were made by gender, age, method and unemployment to the rises and falls in suicide rates in HK over the past 40 years. However the decomposition impact of these factors is not exclusive, as other factors may have contributed to the rate changes during the period. Changes in the methods may affect the distribution of suicide deaths by method in decomposition analysis and this clearly needs more research. The lack of this information is a limitation to interpretation of findings from this research.

Supplementary Information The online version contains supplementary material available at https://doi. org/10.1007/s42379-021-00087-5.

Acknowledgements We are grateful to Census and Statistics Department of the Hong Kong SAR for providing the data of registered suicide deaths and population for present study, and also the constructive comments of the two reviewers.

Author contribution CT YANG and PSF Yip jointly conceived and developed the study proposal. YANG was mainly in charge the data manipulation and analysis. YANG and YIP together wrote this manuscript.

Funding PSFY was supported by General Research Fund grant number GRF106190229 and Humanities and Social Science Prestigious Fellowship (Research Grants Council).

Availability of data and material In supplement section.

Code availability Not applicable.

Declarations

Conflict of interest On behalf of all the authors, the corresponding author states that there is no conflict of interest.

Ethics approval Not applicable.

Consent to participate Not applicable.

Consent for publication All authors read and approved the final version of the manuscript. 


\section{References}

Carroll, J. M. (2007). A concise history of Hong Kong. Rowman \& Littlefield Publishers.

Center for Health Protection (2021). Department of Health, The Government of Hong Kong Special Administrative Region. https://www.chp.gov.hk/en/statistics/data/10/27/111.html. Accessed 24 May 2021

Chan, K. P., Lee, D. T., \& Yip, P. S. (2003). Media influence on suicide media's role is double edged. BMJ, 326(7387), 499.

Chang, S. S., Chen, Y. Y., Yip, P. S. F., Lee, W. J., Hagihara, A., \& Gunnell, D. (2014). Regional changes in charcoal-burning suicide rates in East/Southeast Asia from 1995 to 2011: a time trend analysis. PLoS Medicine, 11(4), e1001622.

Chen, M., \& Yip, P. (2019). Remarriages and transnational marriages in Hong Kong: implications and challenges. Journal of Ethnic and Migration Studies, 46(22), 3059-3077.

Chen, Y. Y., Wu, K. C. C., Yousuf, S., \& Yip, P. S. F. (2012). Suicide in Asia: opportunities and challenges. Epidemiologic Reviews, 34, 129-144.

Chen, Y. Y., Yang, C. T., Cha, E. S., \& Yip, P. S. F. (2020). Quantifying the contributions of age, sex, methods, and urbanicity to the changing suicide rate trends in South Korea, 2001-2016. Social Psychiatry and Psychiatric Epidemiology, 55, 1121-1132.

Chung, R. Y., Yip, B. H. K., Chan, S. S. M., \& Wong, S. Y. S. (2016). Cohort effects of suicide mortality are sex specific in the rapidly developed Hong Kong Chinese population, 1976-2010. Depression and Anxiety, 33, 558-566.

Dodsworth, J., \& Mihaljek, D. (1997). Hong Kong, China growth, structural change, and economic stability during the transition. Washington: International Monetary Fund.

Hau, K. T. (1993). Suicide in Hong Kong 1971-1990: age trend, sex ratio, and method of suicide. Social Psychiatry and Psychiatric Epidemiology, 28(1), 23-27.

Hong Kong Jockey Club Centre for Suicide Research and Prevention. (2020). 1981-2019 Hong Kong Suicide Statistics. https://csrp.hku.hk/statistics/. Accessed 1 Aug 2020

Hong Kong Monthly Digest of Statistics. (2017). Hong Kong population projections 2017-2066. Hong Kong: Census and Statistics Department.

Kaiman, J. (2014). Hong Kong's umbrella revolution-the Guardian briefing. https://www.theguardian. com/world/2014/sep/30/-sp-hong-kong-umbrella-revolution-pro-democracy-protests. Accessed 1 Aug 2020

Kan, G., \& Ma, Z. D. (2008). Examining cross-border marriages in Hong Kong since its return to China in 1997. Population Space and Place, 14(5), 407-418.

Kuroki, M. (2010). Suicide and unemployment in Japan: evidence from municipal level suicide rates and age-specific suicide rates. The Journal of Socio-Economics, 39(6), 683-691.

Lai, C. C. S., Law, Y. W., Shum, A. K. Y., Ip, F. W. L., \& Yip, P. S. F. (2020). A community-based response to a suicide cluster. Crisis, 41(3), 163-171.

Lam, W. M., Lui, P. L. T., \& Wong, W. (2012). Contemporary Hong Kong government and politics (2nd ed.). Hong Kong University Press.

Law, C. K., Yip, P. S. F., \& Caine, E. (2011). The contribution of charcoal burning to the rise and decline of suicides in Hong Kong from 1997-2007. Social Psychiatry, 46(9), 797-803.

Law, C. K., Yip, P. S. F., Chan, W. S. C., Fu, K. W., Wong, P. W. C., \& Law, Y. W. (2009). Evaluating the effectiveness of barrier installation for preventing railway suicides in Hong Kong. Journal of Affective Disorders, 114(1-3), 254-262.

Lee, S. H. (2006). SARS in China and Hong Kong. Nova Science Publishers.

Lin, J. J., Chen, L. H., Huang, S., \& Lu, T. H. (2008). Problems in estimating the number of suicides by charcoal burning in Taiwan. Journal of Epidemiology and Community Health, 62(6), 566.

Ma, N. (2007). Political development in Hong Kong: State, political society, and civil society. Hong Kong: Hong Kong University Press.

Moksony, F. (1990). Ecological analysis of suicide: problems and prospects. In L. David (Ed.), Current concepts of suicide (pp. 121-138). Philadelphia: Charles Press.

National Cancer Institute. (2020). Division of Cancer Control \& Population Sciences. https://surveillan ce.cancer.gov/joinpoint/. Accessed 1 Aug 2020

Ni, M. Y., Yao, X. I., Leung, K. S. M., Yau, C., Leung, C. M. C., Lun, P., Flores, F. P., Chang, W. C., Cowling, B. J., \& Leung, G. M. (2020). Depression and post-traumatic stress during major social unrest in Hong Kong: a ten-year prospective cohort. The Lancet, 395(10220), 273-284. 
Retherford, R. D., \& Cho, L. J. (1973). Comparative analysis of recent fertility trends in East Asia. Washington: East-West Center.

SAS Institute Inc. (2016). Base SAS 9.4 procedures guide-statistical procedures (6th ed.). Cary: SAS Institute Inc.

Task Force on Land Supply. (2018). Land for Hong Kong: Our Home, Our Say! https://www.legco.gov. hk/yr17-18/english/panels/dev/papers/dev20180529-booklet201804-e.pdf. Accessed 1 Aug 2020

Teo, E., \& Fu, Kw. (2021). A novel systematic approach of constructing protests repertoires from social media: comparing the roles of organizational and non-organizational actors in social movement. Journal of Computational Social Science. https://doi.org/10.1007/s42001-021-00101-3

The Government of Hong Kong Special Administrative Region. (2020). Sino-British Negotiations and the Sino-British Joint Declaration. https://www.basiclaw.gov.hk/tc/index/index.html. Accessed 1 Aug 2020

Wong, H. (2016). The impact of globalization: marginalization of Hong Kong labour. Asian Exchange, 16(1), 74-85.

Wong, K., \& Yeung, M. (2019). Population ageing trend of Hong Kong. Hong Kong: Office of the Government Economist-Economic Letter 2019/02.

World Health Organization. (1978). The ninth revision of the international classification of diseases and related health problems (9th ed.). WHO.

World Health Organization. (1992). The tenth revision of the international classification of diseases and related health problems (10th ed.). WHO.

World Health Organization. (2020). Global heath and aging. https://www.who.int/ageing/publications/ global_health.pdf. Accessed 1 Aug 2020

Wu, K. C. C., Chen, Y. Y., \& Yip, P. S. F. (2012). Suicide methods in Asia: implications in suicide prevention. International Journal of Environmental Research and Public Health, 9(4), 1135-1158.

Yip, P. S. (1997). Suicides in Hong Kong, 1981-1994. Social Psychiatry Psychiatric Epidemiology, 32(5), 243-250.

Yip, P. S. F., \& Chau, P. H. (2020). Physical distancing and emotional closeness amidst COVID-19. Crisis, 41(3), 153-155.

Yip, P. S. F., Law, C. K., Fu, K. W., Law, Y. W., Wong, P. W. C., \& Xu, Y. (2010). Restricting the means of suicide by charcoal burning. British Journal of Psychiatry, 196(3), 241-242.

Yip, P. S. F., Law, C. K., \& Law, Y. W. (2003). Suicide in Hong Kong: epidemiological profile and burden analysis, 1981 to 2001. Hong Kong Medical Journal, 9(6), 419-426.

Yip, P. S. F., \& Zheng, Y. (2021). The impact of suicide on life expectancy: a Hong Kong experience. Crisis, 42(2), 107-113. https://doi.org/10.1027/0227-5910/a000695

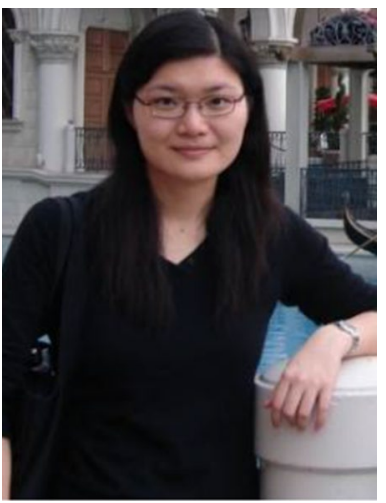

Chi-Ting Yang is a seasoned data scientist with 15 years of experience from academia and commercial sector, whereas 10 years served as an analytical lead at software vendor of SAS Institute Ltd specializing in data analytics, data management, business intelligence and big data solutions. She has more than 10 years of training experiences in SAS programming, statistics, data analytics and big data courses at tertiary level with good feedback from attendees. She offers technical consultation in data-driven technologies, advanced analysis solutions and compliance management. Her publications on the topic of suicide research \& prevention and alcohol pharmacokinetics can be found in the peer-reviewed international journals. Dr Yang obtained her PhD, MPhil and B. Social Science degrees in Statistics (Hon) from The University of Hong Kong. 


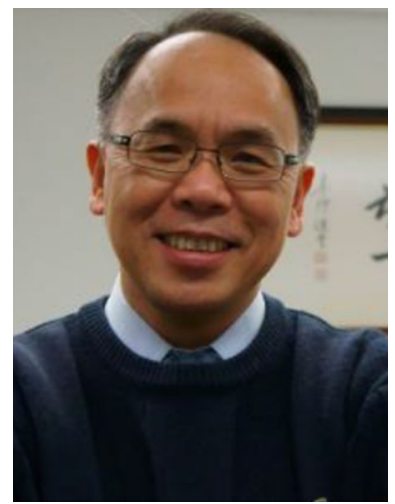

Paul Siu Fai Yip is the chair professor of population health at the Department of Social Work and Social Administration, the Associate Dean (Research) at the Faculty of Social Sciences and the founding director of the Centre of Suicide Research and Prevention at the University. He has led two Chief Executive Community Project on Poverty Alleviation (2012-2017) and Suicide Prevention (20022006). His mission is to identify and develop good practices to promoting wellness in the population. His monograph is on Understanding Poverty in Hong Kong (香港貧穹問題真相) has been awarded the 11th Hong Kong Book Prize, 2018. He is the Chair of the Research Committee of the Family Planning Association of Hong Kong (2012-present). He served as the Secretary General of Asian Population Association (2014-2017), the interim Chairperson of the East and South East Asia and Oceania Region of the International Planned Parenthood Federation (2018-2019), the chairman of the committee of preventing students' suicide (2016) and a member of the Steering Committee on Population Policy (2013) and an associate member of the Central Policy Unit of the HKSAR Government (2012-2014). He is a recipient of the Australia-China Alumni Award for Research in 2019; a medal of honor from the Hong Kong SAR Government in 2017; the Stengel Research Award from the International Association of Suicide Prevention in 2011, outstanding supervisor and researcher of the University of Hong Kong in 2010-11 and 2009-2010 respectively; distinguished alumni of La Trobe University. He has published more than 500 research papers relating to population health. 